\title{
Representación, significado e interacción en el acoso escolar juvenil ${ }^{*}$
}

\author{
Alejandra Patricia Gómez Cabrera' \\ Universidad Nacional Autónoma de México (México)
}

Recibido: febrero 12 de 2016. Revisado: marzo 10 de 2016. Aceptado: mayo 24 de 2016

Referencia format APA: Gómez-Cabrera, A. P. (2016). Representación, significado e interacción en el acoso escolar juvenil. Rev. Guillermo de Ockham, 14(2), 93-101. doi: http://dx.doi.org/10.21500/22563202.2426

\section{Resumen}

El acoso escolar es una problemática que trasciende la violencia física y cuyas implicaciones afectan la convivencia de un modo sutil e implícito, en el que los alumnos son marginados a nivel de su afectividad e identidades. Comprender el acoso escolar como una forma de interacción fundada en el hostigamiento al que dan lugar los procesos de estigmatización y asimetrías emocionales entre alumnos, es una propuesta que trasciende la óptica de la mayoría de los trabajos que desde el enfoque comunicacional abordan el estudio de la violencia suscitada en los contenidos mediáticos, las políticas gubernamentales y el crimen organizado. Esta investigación es producto de un ejercicio de redes semánticas aplicado a 143 alumnos del Colegio de Ciencias y Humanidades, Universidad Nacional Autónoma de México, con el objetivo de comprender el significado que los alumnos dan al acoso escolar y sus implicaciones en la convivencia cotidiana a nivel de la afectividad y la identidad.

Palabras clave: Representación, estigma, asimetría emocional y acoso escolar

\section{Representations, meaning and interaction in youth bullying}

\section{Abstract}

Bullying is a problem that transcends physical violence and whose implications affect the coexistence of a subtle and implicit way, in which students are marginalized to the level of their emotions and identities. Understand bullying as a form of interaction based on the harassment leading processes of stigmatization and emotional asymmetry between students, is a proposal that transcends the perspective of most of the work, from the communication approach, dealing with the study provoked violence from the media content and government policies. This research is the product of an exercise in semantic networks applied to 143 students of the Colegio de Ciencias y Humanidades, Universidad Nacional Autónoma de México, with the goal of understanding the meaning of the students give the bullying and its impact on everyday living to level of emotion and identity.

Keywords: Representation, stigma, emotional asymmetry and bullying

\footnotetext{
* Este artículo es producto de la investigación Interacción social y violencia simbólica: una mirada desde el acoso escolar juvenil, llevada a cabo para obtener el grado de doctora en Ciencias Políticas y Sociales en la Universidad Nacional Autónoma de México (Unam). El trabajo de campo en el que se centra el estudio, se aplicó a una muestra de 143 alumnos del Colegio de Ciencias y Humanidades, Plantel Sur, Unam, ambos turnos, durante los meses de agosto a diciembre del 2015.

1. Candidata al grado de Doctora en Ciencias Políticas y Sociales con orientación en Ciencias de la Comunicación por la Universidad Nacional Autónoma de México. Miembro de la Asociación Latinoamericana de Estudios en el Discurso. Correo electrónico: patriciagomez444@hotmail.com. Dirección Postal: Rosa de Oro 43, Col. Molino de Rosas, C.P. 01470 Delegación Álvaro obregón México, Distrito Federal
} 


\section{Representação, significado e interação no assédio escolar juvenile}

\section{Resumo}

O assédio escolar é uma problematica que transcende violência física e cujas implicaçóes afetam a oexistência de uma maneira sutil e implícita no qual os alunos são marginalizados ao nível de sua afetividade e identidades. Compreender o assédio escolar como uma forma de interaçáo com base no resultado perseguiçáo ao em processos de estigmatização e desequilíbrios emocionais entre estudantes, é uma proposta que transcende a perspectiva da maioria dos trabalhos que desde o abordagem comunicacional lidar com o estudo da violência provocou no conteúdo de mídia, políticas governamentais e ao crime organizado. Esta pesquisa é produto de um exercício de redes semânticas aplicado a 143 estudantes da faculdade de ciências e Humanidades da Universidade Nacional Autônoma do México, com objetivo de compreender o significado que os alunos dão ao assédio escolar e suas implicaçôes na convivencia cotidiana ao nível da afectividade e a identidade.

Palavras-chave: Representação, estigma, assimetria emocional, assédio escola

\section{Introducción}

El enfoque microsocial es uno de los más relegados en las investigaciones en comunicación; sin embargo, categorías como la identidad y la afectividad permiten un conocimiento más profundo de la interacción y de los procesos de socialización en ella inmersos. En este contexto, enfoques como el interaccionismo simbólico (Mead, 1973) y las representaciones sociales (Moscovici, 1979 y Abric, 2001) han orientado sus investigaciones al análisis de la construcción de sentido en el ámbito situacional y al estudio de la persona como eje explicativo de lo social.

Por su parte, en el ámbito de la violencia, el estigma y la asimetría emocional son prácticas que cuentan con cierto nivel de aceptación social, pues en lugar de utilizar la agresión física como un modo de dominio, recurren a la coacción presente en el dinero y el prestigio. Desde esta óptica, la violencia no implica ya el suplicio público, en términos de Foucault, sino un ataque limitado a espacios más privados.

La violencia sigue existiendo, pero a través de un cuidado estratégico que impide "cualquier paso en falso que pueda disminuir el valor de quien la ha realizado y rebajar su posición. El hombre se vuelve dueño de sus gestos, disimula sus malas intenciones y reprime sus pasiones" (Elias, 2008, p. 575).

Bourdieu (2000), define a estas expresiones como violencia simbólica. "Lo que llamo violencia simbólica, violencia amortiguada e invisible para sus propias víctimas, se ejerce esencialmente a través de los caminos puramente simbólicos de la comunicación o, más exactamente, del desconocimiento, del reconocimiento y del sentimiento" (p.11).

Al trascender el comportamiento inmediato, el estigma y la asimetría emocional se sitúan en el nivel latente de la convivencia, en la rutina del encuentro, por lo que es posible naturalizar sus expresiones y disimular la violencia en ellas contenida (Acevedo \& Samacá, 2012). La violencia, por consiguiente, ya no implica un incidente, sino un proceso por medio del cual se expresan las diferencias sociales en cuanto desigualdades.

Este trabajo, por lo tanto, se centra en comprender cómo el acoso escolar constituye una manifestación de la violencia simbólica, sobre la base de entender el concepto de acoso escolar como el comportamiento orientado a dañar al otro a través de "episodios repetidos de violencia contra un alumno en particular, por lo que la agresión se hace sistemática, deliberada y reiterada" (Mendoza, 2013, p.7).

Los convenios instaurados en las relaciones interpersonales y en los contextos de pertenencia grupal, configuran las dinámicas de violencia allí producidas. Por ello, el acoso escolar solo puede comprenderse en el marco de un proceso de interacción comunicativa mediante redes lingüísticas en las que la retroalimentación permanente entre sus protagonistas configura sus personalidades y con ello, los acuerdos de convivencia entre ellos.

\section{Método}

Para el análisis de la representación social, se aplicó en primer lugar un ejercicio de asociación libre de palabras a 143 alumnos del Colegio de Ciencias y Humanidades, 
Plantel Sur, a quienes se les pidió anotaran y jerarquizaran las primeras cinco palabras evocadas a partir de la expresión inductora "acoso escolar". La muestra de alumnos estuvo integrada por un $51 \%$ de hombres y $49 \%$ de mujeres. El $55 \%$ de los estudiantes estaba inscrito en el turno matutino y el $45 \%$ en el vespertino. El $33 \%$ cursaba el primer ańo escolar, el $42 \%$ el segundo y el $25 \%$ el tercero. La edad de los alumnos osciló entre los catorce y los diecinueve años. Cabe mencionar que el estudio se llevó a cabo durante el ciclo escolar 2014. ${ }^{2}$

"El carácter espontáneo y la dimensión proyectiva de la asociación libre permiten actualizar los elementos implícitos y latentes que estructuran el universo semántico del objeto estudiado" (Abric, 1994, p. 60). Lo anterior, para comprender las formas como los alumnos conciben el acoso escolar, así como las acciones y los juicios que guían este tipo de prácticas.

Con base en las propuestas de Abric (1994) y Moscovici (1979), se desarrolló la siguiente metodología de análisis:

1. Se diseñó una base de datos con el número total de palabras asociadas la noción de acoso escolar. Se agruparon en un solo término las palabras con un significado similar, para que solo permanecieran aquellos ítems con un sentido distinto. Posteriormente, se organizaron las palabras resultantes en categorías de acuerdo con los criterios de referencia propuestos por la investigación.

2. Con base en los ejes de análisis, la frecuencia y el rango de aparición de las palabras evocadas, se sistematizó la representación mediante sus elementos constitutivos, a saber, el núcleo figurativo y la zona periférica. A partir de ello, se identificaron los recursos usados por los alumnos para comprender el acoso escolar.

\section{Resultados}

\section{El significado social del acoso escolar}

En esta parte del análisis se enlistan el contenido de las categorías de las que se valen los alumnos para sustentar su experiencia en torno al acoso escolar. Este es un primer acercamiento, que "no expresa todo el contenido de la representación, simplemente nos autoriza a comprobar la existencia de una organización subyacente al conteni- do. "Descubrir la orientación global en relación con el objeto de la representación social y la toma de posición directa" (Moscovici, 1979, p. 47) de los alumnos, en cuanto individuos y grupos sociales que retroalimentan esta representación.

Los 143 alumnos entrevistados asociaron un total de setenta y tres términos distintos al acoso escolar. Una vez se depuraron las palabras con un significado parecido, se identificó un universo representacional semejante entre los alumnos, pues entre uno y otro prevalecen los mismos significados para definir esta práctica.

Posteriormente, las palabras expresadas se agruparon en categorías en virtud del sentido temático que comparten y a la relación que mantienen con el contenido de los ejes teóricos de esta investigación: la violencia física, la violencia simbólica, el estigma, la asimetría emocional, la exclusión y las consecuencias de la violencia.

Los términos contenidos se asocian con valores, ideas y prácticas particulares; las categorías aportan un marco para establecer un rango de elementos materiales, tendencias personales y estilos conductuales. Estos, a su vez, se entienden como indicadores [del acoso escolar] y aportan los recursos que usan los individuos para expresarse (Castorina, 2003, p. 41).

Este corpus de conceptos organizados en categorías sintetiza el panorama semántico del que se valen los alumnos para organizar sus experiencias en torno al acoso escolar, facilitando así la comprensión y dándoles sentido a los hechos que comparten (Figura 1).

Para los alumnos entrevistados, la violencia es un concepto central para comprender el acoso escolar, comoquiera que implica una forma de abuso que expresa la intención perversa e injusta de querer dañar, poner en peligro o evidenciar de una forma negativa al otro. También destaca el hecho de percibir el acoso como una respuesta impulsiva, una forma de explotar ante una situación que al salir del control de sí mismo repercute en él y en su entorno.

En relación con la violencia simbólica, el acoso escolar es percibido mediante prácticas como los insultos, la discriminación y la violencia emocional, principalmente. $\mathrm{Al}$ respecto, los alumnos encuestados mencionaron a las agresiones verbales y las burlas presentes en un $38 \%$ de los casos, como una de las prácticas más frecuentes de

2. En México, en el 2014, se registraron, por lo menos dos casos trágicos de acoso escolar que terminaron con la vida de los alumnos agredidos. En Tamaulipas, en el mes de mayo, dos menores murieron por lo golpes propiciados por compańeros de escuela. Una de ellos, en la secundaria Ignacio Zaragoza y el otro, en la secundaria Eleázar Gómez. Estos acontecimientos llamaron la atención de los medios de comunicación y las autoridades estatales y federales. De allí que la mayoría de las estrategias en materia de acoso escolar se hayan iniciado en este año. 


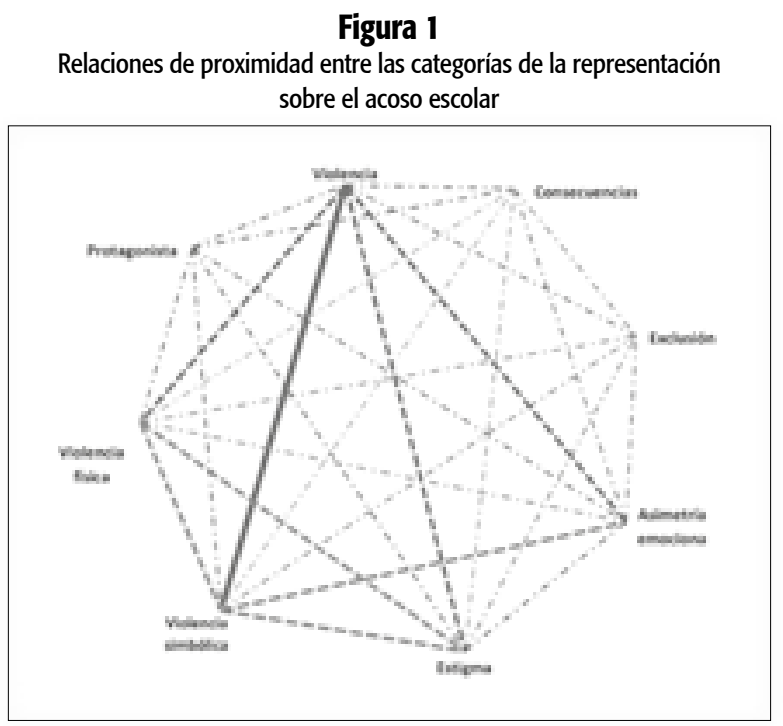

Fuente: Elaboración propia.

acoso escolar. Por su parte, el $46 \%$ dijo que un alumno agrede por miedo, mientras que el $68 \%$ mencionó que el agresor aprovecha el miedo que le tienen para actuar violentamente.

Los insultos evidencian una forma de ridiculización en la que las palabras son usadas como un medio para estigmatizar, incomodar u obstaculizar la rutina e identidad del otro. La discriminación, por su parte, es una forma de marginar y diferenciar al otro mediante la exclusión que terceras personas ejercen sobre él. Finalmente, la violencia emocional implica una forma de intimidación y desprecio por medio de las emociones que identifican la otredad y la ponen en un estado de indefensión debido a sus sentires.

Aunque en menor medida, la falta de ética en la convivencia rutinaria también es vista por los alumnos como una manifestación de la violencia. La falta de valores, los estereotipos y los prejuicios, la manipulación y la hipocresía, refieren prácticas que al fomentar la desigualdad afectan la integridad sociopersonal de un alumno y con ello devienen en manifestaciones de acoso escolar. Al respecto, un 37,3\% de los alumnos encuestados mencionó que la falta de valores es el principal problema que genera violencia entre los jóvenes.

En el ámbito de la asimetría emocional, destaca el miedo como la principal emoción a partir de la cual, los alumnos identifican el acoso escolar. También señalan a la tristeza como otra de las emociones frecuentes, especialmente en la identidad de la víctima. En este sentido, el miedo, 38,2 \% y la tristeza, 21,9\% destacan como los principales afectos para identificar a la víctima. Por su parte, el poder, 21,9\%, la fuerza, 20,5 \% y la alegría, $13,7 \%$ son los principales afectos atribuidos al agresor.
En el análisis del acoso escolar a partir del referente del estigma, los alumnos ubican la ridiculización como el principal mecanismo de estigmatización en la convivencia rutinaria. Las burlas son utilizadas como herramienta de diversión y pasatiempo juvenil en los que algunos alumnos son utilizados como una especie de bufones para divertir al resto del grupo. Asimismo, las burlas se usan como un medio de diferenciación marginal del otro y como una estrategia mediante la que el agresor salvaguarda la apariencia para evitar ser estigmatizado por el uso de la violencia como medio de control y jerarquía.

Por su parte, los alumnos entrevistados consideran que los golpes son la principal manifestación de violencia física. En menor medida, distinguen otras formas de agresión física tales como las peleas, los disturbios y el robo. En relación con las consecuencias de la violencia, los alumnos entrevistados consideran que el dolor es el principal resultado del acoso escolar. Al respecto, el 28,2 $\%$ de los encuestados considera que la violencia afecta negativamente su autoestima. También hay una tendencia a relacionar las consecuencias de la violencia con los problemas, lo cual refuerza la connotación negativa de los alumnos en relación con el acoso escolar.

No obstante, es importante referir que aun cuando hay una conciencia social sobre el dolor individual que causa el acoso escolar en el agredido, no existe una conciencia sobre el daño causado a terceros. La conciencia acerca del dolor se ubica en el plano del corto plazo; no hay evidencias de que ubiquen consecuencias a mediano o largo plazo. Finalmente, llama la atención que el aprendizaje en torno a las consecuencias de la violencia se sitúa en el último peldaño de esta categoría, incluso por debajo de la venganza. Ello explica el porqué de los ciclos interminables de violencia frente a este fenómeno.

En los últimos peldaños de repetición se sitúan las categorías protagonistas de la violencia y exclusión. El agresor, la víctima y los espectadores, son los protagonistas de la violencia mencionados por los alumnos. Sin embargo, el hecho de que los mencionen en tan pocos momentos explica su propensión a comprender el acoso escolar como una forma de violencia en abstracto; es decir, entre los estudiantes hay una dificultad para darle rostro a la agresión, posiblemente como una manera de evitar el compromiso que significa hacerle frente a dicha problemática.

La escasa frecuencia con la que los alumnos expresaron la exclusión como una forma de acoso escolar, deja entrever que para ellos el rechazo y el aislamiento, pocas veces representan formas de violencia en la escuela. Mientras las víctimas de violencia reconocen las consecuencias

$96<$ Universidad de San Buenaventura, Cali - Colombia 
específicas de la exclusión en el mismo grado que las consecuencias de la violencia física, para el resto de los alumnos, la exclusión sólo es uno medio que aumenta las posibilidades de ser agredido.

\section{Discusión}

\section{Representación e interacción en el acoso escolar}

El análisis de una representación requiere una doble identificación en la que, además del contenido de las categorías, estas se organizan con base en la lógica constitutiva de la representación social: el núcleo figurativo y la zona periférica próxima y distante.

La sistematización de estos elementos permite explorar las formas como los sistemas sociocognitivos a los que da lugar la representación se organizan, jerarquizan y complementan internamente, así como su funcionamiento externo a través de la toma de decisiones, las reglas y las prácticas mediante las cuales los alumnos guían y determinan las forma de interacción en un contexto de acoso escolar (Figura 2).

Figura 2

Sistematización de la representación sobre acoso escolar

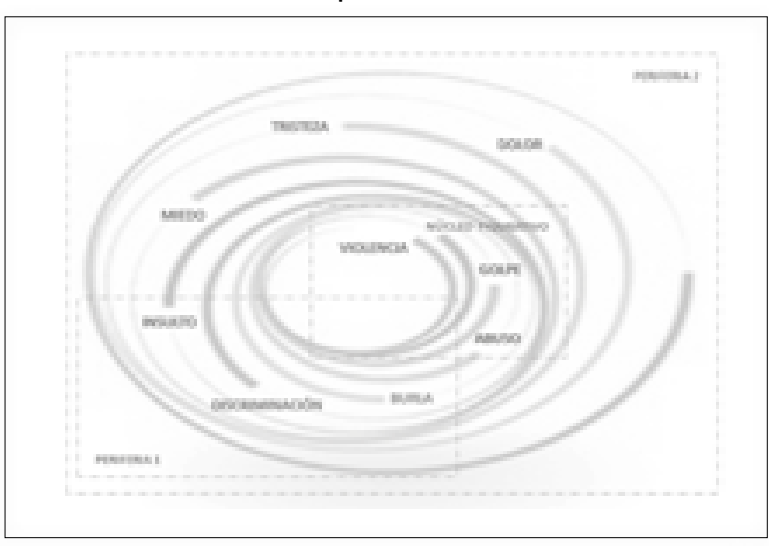

Fuente: Elaboración propia.

\section{La construcción de estereotipos alrededor del acoso escolar}

El núcleo figurativo es la parte de la representación más estable, pese a las transformaciones del devenir sociohistórico y cultural. Sus elementos ayudan a simplificar y entender la realidad desde un punto de vista comunitario en el que las experiencias individuales y rutinarias son incorporadas a estándares más estructurales.

Para Abric (2001)

el núcleo central está relacionado con las condiciones históricas e ideológicas. Es la base común que define la homo- geneidad de un grupo. Desempeña un papel esencial en la estabilidad y la coherencia de la representación y garantiza su perennidad y conservación en el tiempo (p. 26).

Las palabras asociadas al concepto de acoso escolar mencionadas con una alta frecuencia y aparecidas en los primeros lugares de evocación constituyen el núcleo figurativo de la representación. Los términos violencia, golpe y abuso conforman el contenido del núcleo figurativo de la representación sobre acoso escolar evidenciada por los alumnos entrevistados.

La estabilidad y generalización que caracteriza a los conceptos del núcleo figurativo expresa, a su vez,

un momento decisivo en la formación de actitudes prejuiciosas y estereotipos, lo que implica: una reacción de los individuos ante un objeto dado desde afuera, acabado, independientemente del actor social, de su intención o sus características, y un anuncio interiorizado de la futura acción (Moscovici, 1979, p. 30).

El prejuicio y el estereotipo se corresponden con los procesos de aprendizaje de las creencias y las normas sociales a través de las cuales se concibe el acoso escolar, por lo cual sirven a los alumnos como herramientas cognitivas, conductuales y afectivas para adaptarse a los patrones de convivencia en este entorno

El estereotipo es la imagen mental fundada en la generalización de un hecho o grupo social en específico. De modo que en el estereotipo se retoman las características más significativas de un hecho y se imprimen al objeto tratado como atributos que condensan su totalidad. "Los aspectos funcionales y dinámicos que poseen los estereotipos como simplificadores de un entorno complejo, producen una preparación para la percepción de comportamientos y características con base en la información precedente" (Ungaretti, 2013, p. 16).

Identificar el acoso escolar como un tipo de violencia en el que prevalecen las agresiones físicas, es un estereotipo recurrente entre los alumnos para simplificar la amplia gama de prácticas, protagonistas y condicionantes del acoso escolar. Aunque este no se limita a la violencia física, este estereotipo facilita la comprensión y ayuda a los alumnos a sobrellevar una problemática social que por sí misma es más compleja.

"La sustancia simbólica de la realidad, elaborada por los individuos al intercambiar sus modos de ver, tiende a modelarlos recíprocamente. Los estereotipos nunca se hallan aislados, siempre corresponden a sus relaciones con el mundo" (Moscovici, 1979, p. 32). De allí que el este- 
reotipo opera ligado con el prejuicio. La sistematización de la realidad a través del estereotipo es solo un preámbulo de las condicionantes que orientarán la interacción entre alumnos en situaciones específicas de socialización.

"El prejuicio hace referencia a lo infundado de un juicio y al tono afectivo que lo acompaña" (Allport, 1968, p. 21), determinando de esta manera actitudes y valores diferenciados para posicionarse, justificar y evaluar la realidad escolar en torno a prácticas consideradas formas de acoso escolar.

$\mathrm{Al}$ respecto, los estereotipos operan como factores desencadenante de procesos evaluativos de la realidad social que conducen a su organización diferenciada y jerarquizada. A la idea de que el acoso escolar es un tipo de violencia en el que prevalecen las agresiones físicas corresponde, a su vez, el prejuicio de valorar estos modos de interacción como manifestaciones de abuso.

Para los alumnos entrevistados, el abuso es la disolución al contrato de convivencia, a las tradiciones o a las normas establecidas por los alumnos pertenecientes a cierto grupo y encaminan a la estigmatización y a la exclusión de aquellos que contradicen dichas reglas, porque con sus prácticas evidencian formas de acoso escolar que repercuten en el ánimo e integridad de los otros.

El individuo difícilmente puede ignorar lo que piensan los demás sobre la mayoría de las cuestiones que piden una respuesta de su parte. Al existir semejanzas interindividuales respecto a la respuesta evaluativa inmediata o a la intención de actuar en relación con un objeto determinado (Moscovici, 1985a, p. 118).

Tanto las agresiones físicas como el abuso predisponen a los alumnos a tomar una posición negativa ante estas prácticas, lo que contribuye a diferenciar marginalmente a quienes las protagonizan. Por consiguiente, la agresión física y el abuso son conceptos que, además de identificar de manera per se los principales atributos y categorías del acoso escolar -los cuales no siempre congenian con la realidad- motivan a ideas erróneas en las que, por ejemplo, el acoso escolar es visto como una problemática que solo involucra a los protagonistas directos y deja de lado la responsabilidad que aqueja al resto de la comunidad.

De este modo, las generalizaciones que traen consigo los estereotipos y los prejuicios originan etiquetamientos nocivos hacia los protagonistas directos del acoso escolar, con lo cual en lugar de resolver el problema de raíz dan paso a la marginación y exclusión de los alumnos en él inmersos debido a que pocas veces abarcan sus experiencias personales y el acontecer rutinario de sus voces y vivencias.

\section{Prácticas sociales en torno al acoso escolar}

Los elementos que conforman la periferia de la representación están subordinados al significado y al funcionamiento del núcleo figurativo. Son los aspectos más precisos y detallados de las experiencias que cotidianamente viven los alumnos en torno al acoso escolar. Evidencian la lógica de lo que es normal e inmediato en el ocurrir de la realidad habitual.

Estos elementos están jerarquizados, es decir que pueden estar más o menos cercanos a los elementos centrales: próximos al núcleo, desempeñan un papel en la concreción del significado de la representación, más distantes de él ilustran, aclaran y justifican esta significación (Abric, 1994, p. 23).

Los elementos mencionados frecuentemente, pero que son nombrados en los últimos lugares, constituyen la primera periferia. La burla, la discriminación y el insulto son los elementos de la periferia más cercanos al núcleo figurativo y representan las formas específicas como el acoso escolar es experimentado por los alumnos entrevistados.

"La transformación de un conocimiento indirecto en un concomimiento directo [sentido común], es el único medio de apropiación del universo exterior" (Moscovici, 1979 , p. 35). Esto significa que aún cuando los alumnos señalan que la violencia, los golpes y el abuso son las ideas centrales para entender y explicar el acoso escolar, las burlas, la discriminación y los insultos son las maneras como lo experimentan en el día a día.

En su bagaje explicativo -creencias y actitudes- para referirse al acoso escolar, los alumnos hacen uso de los elementos del núcleo figurativo, a saber, la violencia, los golpes y el abuso, los cuales expresan abstracciones generales que alimentan las categorías que le dan orden y sentido a una realidad compleja y difusa como el acoso escolar.

Mientras tanto, cuando abordan sus propias experiencias de acoso escolar, retoman los elementos de la primera periferia: las burlas, la discriminación y los insultos; conceptos que, a su vez, traducen el universo desconocido en algo familiar. Ello confirma el hecho de que el acoso escolar no solo se asocia con la agresión física, sino también con prácticas más sutiles como la ridiculización del otro mediante los insultos y la discriminación.

En la violencia simbólica, tanto el prejuicio como el estereotipo conducen a la estigmatización. El estigma es un elemento por medio del cual el prejuicio y el estereotipo adquieren corporalidad y sirven como una etiqueta que tilda al otro de extraño frente a las cualidades identitarias

$98<$ Universidad de San Buenaventura, Cali - Colombia 
de un grupo o comunidad. "El estigma es un atributo que vuelve diferente a un individuo frente a los demás y lo convierte en alguien menos. Un atributo de esta naturaleza produce en los demás un descrédito amplio" (Goffman, 2008, pp. 14-15).

Aunque la agresión física es un recurso al que con frecuencia recurren los alumnos en sus discursos para referirse al acoso escolar, ello no ocurre con la misma magnitud en sus prácticas cotidianas, debido a que la violencia física trae consigo altos niveles de estigmatización y exclusión social, por lo que estas prácticas son evitadas entre la comunidad estudiantil. Al respecto, Collins (2011) explica:

The tendency to become entrained in each other's rhythms and emotions means that when the interaction is at cross purposes (an antagonistic interaction) people experience a pervasive feeling of tension. For this reason, violence is difficult to carry out, not easy. Those individuals who are good at violence are those who have found a way to circumvent confrontational tension/fear, by turning the emotional situation to their own advantage and to the disadvantage of their opponent (p. 19).

En lugar de confrontar la tensión difundida por la estigmatización inherente a las agresiones físicas frente al resto de la comunidad, la mayoría de los alumnos opta por mecanismos más sutiles de acoso entre los que destaca la ridiculización.

La ridiculización es un mecanismo de estigmatización que utiliza las burlas como medio para insultar y discriminar a ciertos alumnos. A pesar de que es una práctica de acoso escolar, no es vista así por la mayoría de los estudiantes en virtud a que este mecanismo mantiene la consonancia emocional entre ellos al acentuar el sentido irónico de estas prácticas por encima del violento.

Las interacciones sociales están pautadas por experiencias emocionales que crean realidades compartidas y robustecen a los individuos y a sus grupos de pertenencia de sentimientos de apoyo, protección y empatía social. Los episodios de diversión y socialización que traen consigo las burlas, son un elemento clave para compartir emociones, "una energía infusa de moralidad, para que sus actos parezcan de la máxima importancia y valor. La participación grupal en la interacción infunde en sus miembros una fuerza emocional que los transforma en seguidores entusiastas" (Collins, 2009, p. 61).

Independientemente de que algunas burlas deriven en la marginación, la energía emocional que colma al grupo será suficiente para justificar y legitimar estas prácticas como formas de entretenimiento. Los alumnos burlados que evidencien la violencia a la que son expuestos, al romper con los patrones de diversión y energía emocional del grupo serán estigmatizados y excluidos. Para ellos, quedarán bajas dosis de energía emocional y ausencia de solidaridad.

De este modo, por medio de la ridiculización se deja de lado la confrontación emocional de las agresiones físicas. No obstante, se naturalizan otras prácticas violentas en el acontecer rutinario de la convivencia. Pese a que algunas burlas constituyen insultos y prácticas discriminatorias, son abordadas por los entrevistados como insumos de socialización y diversión, con lo cual se enmudecen las vivencias de las víctimas tras el velo del encubrimiento e invisibilización.

Ahora bien, los elementos evocados con menor frecuencia y en los últimos lugares de la lista conforman la segunda periferia de la representación social. Es aquella que está más alejada del núcleo figurativo, razón por la cual es más propensa a cambiar dependiendo del contexto específico en el que se advierte una situación de acoso escolar.

En esta periferia se localizan el miedo, la tristeza y el dolor. Tales atributos refieren aspectos de la asimetría emocional y consecuencias del acoso escolar que devienen de un proceso arraigado en el prejuicio, el estereotipo y el estigma (Figura 3).

\section{Figura 3}

Concepción del acoso escolar con base en el contenido de las zonas periféricas

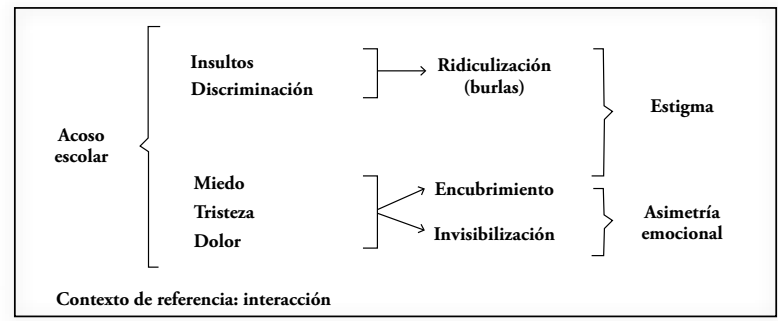

Fuente: Elaboración propia.

El dolor es percibido como consecuencia del acoso escolar y característica identitaria de la víctima. Como consecuencia del acoso, el dolor es una condicionante de la angustia y la indefensión como principales atributos identitarios de la víctima.

Para los alumnos entrevistados, es evidente que las víctimas de violencia suelen perpetuarse en su rol, debido a que no hay una posibilidad inmediata que cambie la relación que existe entre las características identitarias en su perfil, las elegidas para la agresión y las percibidas como consecuencias de la agresión (Figura 4). 
Figura 4

La asimetría emocional en la prolongación del acoso escolar

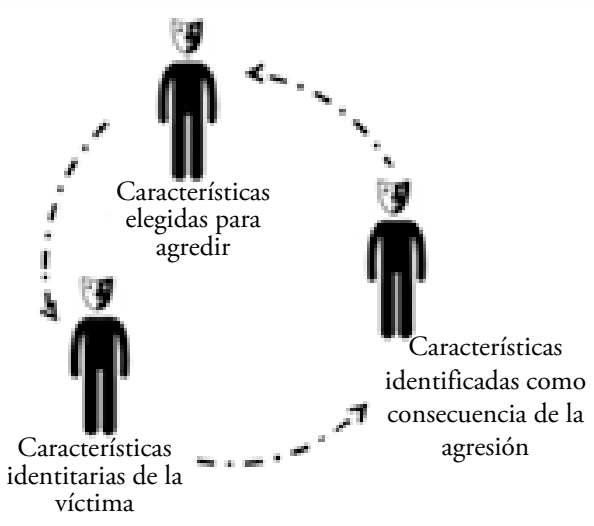

Fuente: Elaboración propia.

Además, estos atributos dejan ver que en la vida diaria el acoso escolar expone con mayor énfasis a la víctima en relación con cualquier protagonista de este fenómeno social. No obstante, al ubicarse en la segunda periferia, estos elementos acogen las mayores transformaciones según la experiencia específica vivenciada por los alumnos.

Esto quiere decir que en el imaginario de los estudiantes aún cuando existe un patrón identitario para ubicar a las víctimas de acoso -la indefensión-, hay alumnos que pueden ser víctimas de agresión a pesar de no incorporar en su identidad algunos de estos sentires. El agresor, por su parte, puede agredir independientemente de que sus víctimas cumplan con estas características.

Asimismo, aunque los alumnos perciben que entre las consecuencias del acoso escolar el dolor y la asimetría emocional son las más habituales, saben que las consecuencias pueden variar de acuerdo con varios factores, entre los que destacan el tipo de agresión vivida y los acuerdos de convivencia establecidos entre los participantes.

\section{Conclusión}

En conclusión, es posible advertir que la violencia mantiene fuertes relaciones proximales con la mayoría de las categorías de la representación. Esto significa que el acoso escolar, independientemente de su modalidad, refiere una manifestación de violencia para los alumnos, pues los pone en desventaja o peligro debido al maltrato que reciben de otros compañeros.

Con base en la fuerza de las correlaciones que establece la violencia con otras categorías de la representación, también es posible identificar los niveles de percepción de violencia que identifican los alumnos en las principales prácticas de acoso escolar que viven en su cotidianidad. El vínculo más notorio lo mantiene el acoso escolar con la categoría de violencia simbólica, forma de agresión en la que los alumnos también perciben los mayores niveles de agresión.

Como se mencionó anteriormente, en la cotidianidad el acoso escolar se vive a través de prácticas más cercanas a la violencia simbólica. Sin embargo, es importante senalar que los insultos y la discriminación también son las prácticas con mayores niveles de encubrimiento a través de las burlas, estas últimas, percibidas por la comunidad de estudiantes como prácticas con menores niveles de violencia.

Aunque los alumnos entrevistados reconocen que existe más violencia en los insultos y la discriminación, el encubrimiento de estas prácticas mediante la ridiculización, reduce su impacto violento y sus efectos nocivos en la percepción pública. Por ello, las burlas, sin importar su contenido hostil, suelen naturalizarse como herramientas propias de la convivencia rutinaria.

Otro de los vínculos proximales más significativos es entre la violencia y la categoría de violencia física. Los golpes son advertidos como la segunda práctica de acoso escolar que causa violencia entre los alumnos. Es posible que si la violencia física fuera más común en su rutina cotidiana, se consideraría como la primera causa de violencia entre los alumnos, no solo por su frecuencia, sino porque los efectos de este tipo de agresiones tienen más visibilidad pública en comparación con los de la violencia física

Con base en esta acepción, los alumnos evalúan negativamente un comportamiento violento cuando los afecta con más frecuencia, aunque sus efectos no sean visibles (tal es el caso de la violencia simbólica) mientras que son más laxos en sus juicios cuando la violencia es menos frecuente, aunque sus efectos sean más visibles, como sucede con la violencia física. Ello indica que para los alumnos, es la cercanía de un hecho violento y no la visibilidad de sus efectos lo que lleva a un juicio negativo en torno al agravio.

La visibilidad de la violencia adquiere un tinte significativo cuando evidencia sus consecuencias en el perfil de la víctima. El miedo y la tristeza son emociones que tienden a evaluar negativamente al alumno agredido en términos de su indefensión ante la experiencia vivida, y dan lugar a otra manifestación de la violencia simbólica conocida como asimetría emocional. 
Ante ello, el agravio físico y el simbólico se retroalimentan mutuamente tanto en la elección como en la permanencia de la víctima frente a dicho estatus, pues quien es agredido físicamente se convierte en blanco de insultos y discriminación a causa del estado de miedo y tristeza que experimenta.

Asimismo, es posible advertir el vínculo entre la violencia simbólica y el estigma. La ridiculización es el mecanismo de estigmatización más importante por la frecuencia con la que los alumnos utilizan las burlas como formas acoso escolar, y al mismo tiempo, como un modo de ocultar la violencia que conllevan prácticas como los insultos y la discriminación.

\section{Referencias}

Abric, J. (2001). Prácticas sociales y representaciones. Coyoacán: México.

Acevedo, A., \& Samacá, G. (2012). Juventud y protesta global hoy: por un análisis retrospectivo. Revista Guillermo de Ockham 10(1), 15-25.

Arauz, R. y Sandoval, A. (coords.). (2003). Tras las vetas de la investigación cualitativa. Perspectivas y acercamientos desde la práctica. México: Instituto Tecnológico y de Estudios Superiores de Occidente.

Allport, G. (1968). La naturaleza del prejuicio (3a ed.) Buenos Aires: Rivadavia.

Banchs, M. (2000). Aproximaciones Procesuales y Estructurales al Estudio de las Representaciones Sociales. Peer Reviewed Online Journal, (9), pp.1-15.

Berger, P. y Luckmann, T. (2008). La construcción social de la realidad. Buenos Aires: Amorrortu.

Bifani-Richard, P. (2004). Violencia, individuo y espacio vital. México: Universidad de la Ciudad de México.

Bourdieu, P. (2000). La dominación masculina. Barcelona: Anagrama.

Calvo, A y Ballester, F. (2013). El acoso escolar: procedimientos de intervención. Sevilla: EOS.

Castorina, J. (comp.). (2003). Representaciones sociales. Problemas teóricos y conocimientos infantiles. Barcelona: Gedisa.

Collins, R. (2009). Cadena de rituales de interacción. Barcelona: Anthropos.

Collins, R. (2008). Violence. A Micro-Sociological Theory. Princeton University Press.

Elias, N. (2008). El proceso de la civilización. Investigaciones sociogenéticas y psicogenéticas ( $3^{\mathrm{a}}$ ed.). México: Fondo de Cultura Económica.
Estévez, E; Ferrer, B; Jiménez, T. (2009). Las Relaciones sociales en la escuela: el problema del rechazo escolar. Psicología educativa. (15), pp.5-20.

Furlan, A. (2012). Reflexiones sobre la violencia en las escuelas. México: Siglo XXI.

Goffman, E. (2004). La presentación de la persona en la vida cotidiana ( $5^{\mathrm{a}}$ ed.). Buenos Aires: Amorrortu Editores.

Goffman, E. (2008a). Estigma. La identidad deteriorada (2a ed.). Buenos Aires: Amorrortu Editores.

Goffman, E. (2008b). Internados: ensayo sobre la situación social de los enfermos mentales. Buenos Aires: Amorrortu Editores.

Gómez, A. (2011). El estigma. La máscara de la comunicación. Tesis de Maestría. Universidad Nacional Autónoma de México.

León, E. (2005). Sentido ajeno. Competencias ontológicas y otredad. Barcelona: Anthropos.

Maturana, H. (1996). El sentido de lo humano. Santiago de Chile: Dolmen Ediciones.

Masini, E. y Galtung, J. (1979). Visiones de sociedades deseables. México: Centro de Estudios Económicos y Sociales del Tercer Mundo.

Mead, G. (1973). Espiritu, persona y sociedad. Desde el punto de vista del conductismo social. Barcelona: Paidós.

Mendoza, B. (2013). Bullying. los múltiples rostros del acoso escolar. México: Pax.

Moscovici, S. (1979). El psicoanálisis, su imagen y su público. Buenos Aires: Huemul.

Moscovici, S. (1985b). Psicología social I. Influencia y cambio de actitudes. individuos y grupos. Barcelona: Paidós.

Olwens, D. (1993). Conductas de acoso y amenaza entre escolares. Madrid: Morata.

Pörhölä, M y Kinney, T. (2010). El Acoso. Contextos, consecuencias y control. Madrid: Aresta.

Ungaretti, J. (2013). El estudio del prejuicio desde una perspectiva psicológica. Cuatro periodos histórico-conceptuales para la comprensión del fenómeno. Calidad de Vida, 8(IV), pp.13-30.

Vergès, P. (1997). Representaciones y determinación social. Fermentum, 7(20), pp.15-30.

Wieviorka, M. (2003). La diferencia. Paris: Plural Editores.

Yang, L; Kleinman, A; Link, B. (Junio, 2007). Cultura y estigma. La experiencia moral. Este Pais. (195), pp.4-15. 